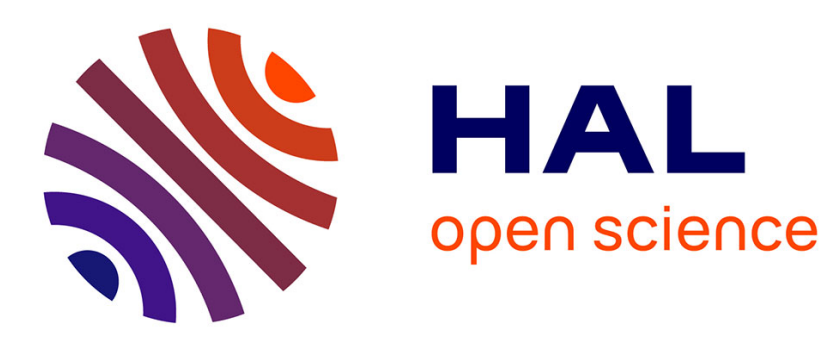

\title{
Confined Water's Dielectric Constant Reduction Is Due to the Surrounding Low Dielectric Media and Not to Interfacial Molecular Ordering
}

\author{
Jean-François Olivieri, James T Hynes, Damien Laage
}

\section{To cite this version:}

Jean-François Olivieri, James T Hynes, Damien Laage. Confined Water's Dielectric Constant Reduction Is Due to the Surrounding Low Dielectric Media and Not to Interfacial Molecular Ordering. Journal of Physical Chemistry Letters, 2021, pp.4319-4326. 10.1021/acs.jpclett.1c00447 . hal-03214193

\section{HAL Id: hal-03214193 \\ https://hal.sorbonne-universite.fr/hal-03214193}

Submitted on 30 Apr 2021

HAL is a multi-disciplinary open access archive for the deposit and dissemination of scientific research documents, whether they are published or not. The documents may come from teaching and research institutions in France or abroad, or from public or private research centers.
L'archive ouverte pluridisciplinaire HAL, est destinée au dépôt et à la diffusion de documents scientifiques de niveau recherche, publiés ou non, émanant des établissements d'enseignement et de recherche français ou étrangers, des laboratoires publics ou privés. 


\title{
Confined Water's Dielectric Constant
}

\section{Reduction Is Due to the Surrounding Low}

Dielectric Media and Not to Interfacial

\section{Molecular Ordering}

\author{
Jean-François Olivieri, ${ }^{\dagger}$ James T. Hynes, ${ }^{\dagger, \dagger}$ and Damien Laage ${ }^{*, \dagger}$ \\ $\dagger$ †ASTEUR, Department of Chemistry, École normale supérieure, PSL University, \\ Sorbonne Université, CNRS, 75005 Paris, France \\ $\ddagger$ Department of Chemistry, University of Colorado, Boulder, CO 80309, USA \\ E-mail: damien.laage@ens.psl.eu
}




\begin{abstract}
Liquid water confined within nanometer-sized channels exhibits a surprisingly low dielectric constant along the direction orthogonal to the channel walls. This is typically assumed to result from a pronounced heterogeneity across the sample: the dielectric constant would be bulk-like everywhere except at the interface, where it would be dramatically reduced by strong restrictions on interfacial molecules. Here we study the dielectric properties of water confined within graphene slit channels via classical molecular dynamics simulations. We show that the permittivity reduction is not due to any important alignment of interfacial water molecules, but instead to the longranged anisotropic dipole correlations combined with an excluded-volume effect of the low-dielectric confining material. The bulk permittivity is gradually recovered only over several nanometers due to the impact of long-range electrostatics, rather than structural features. This has important consequences for the control of, e.g., ion transport and chemical reactivity in nanoscopic channels and droplets.
\end{abstract}

\title{
Graphical TOC Entry
}

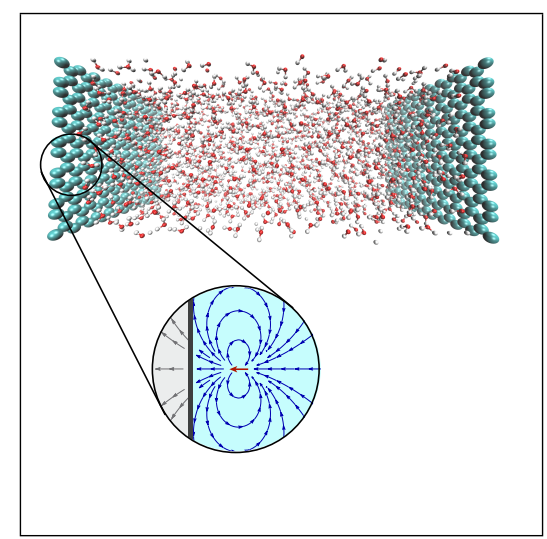


The structural and dynamical properties of liquid water can dramatically change upon confinement within nanometer-sized channels, ${ }^{1,2}$ and one of the properties most spectacularly affected is the water dielectric constant. Numerical simulations have predicted that confinement leads to a strong anisotropy in the dielectric response, ${ }^{3-7}$ and can reduce the $\epsilon_{\perp}$ dielectric constant with respect to the bulk $\epsilon_{b}$ value by several-fold along the direction orthogonal to the channel walls. ${ }^{5,8,9}$ Confirmation was provided by recent atomic-force scanning dielectric microscopy experiments ${ }^{10}$ on liquid water within nanochannels, which revealed that the dielectric constant (as defined in the experiments) exhibits a striking drop from $\epsilon_{\perp} \simeq 80$ to $\epsilon_{\perp} \simeq 2$ when the channel width is decreased from $100 \mathrm{~nm}$ to $1 \mathrm{~nm}$.

The implications of these aspects are of very considerable importance in contexts ranging over such varied areas as energy storage in aqueous supercapacitors, water desalination in nanochannels,${ }^{11}$ ion transport in biological membrane channels ${ }^{12}$ and charge-transfer reactions at electrodes. ${ }^{13}$ In addition - and notably - this interfacial phenomenon is apparently not specific to water: further molecular dynamics simulations ${ }^{6}$ suggested that dielectric constant reduction also applies to both protic and aprotic confined solvents.

The confined water low effective dielectric constant has typically been interpreted ${ }^{3,4,6,10,14-21}$ as arising from an average over distinct regions with very different water properties: bulklike (b) water with $\epsilon_{b} \simeq 80$ in the middle of the slab, in between two extremely low $\epsilon_{i} \simeq 2$ interfacial $(i)$ water layers. In analogy to the so-called electrically 'dead' layer in solid-state capacitors, ${ }^{22}$ the very low $\epsilon_{i}$ interfacial dielectric response was suggested ${ }^{6,10,16,19,21}$ to result from the alignment of interfacial water molecules induced by the channel walls. In this connection, the confined water effective dielectric constant was described by a model involving a series of three capacitors: a bulk layer between two interfacial layers; the model interfacial layer thickness could then be adjusted to yield the same dielectric constant decrease with decreasing channel width as observed in experiments and simulation. ${ }^{10,14,15,23,24}$

However, a source of major difficulty with this model and its interpretation of the reduced $\epsilon_{\perp}$ in confinement is the postulate that interfacial water exhibits a vanishingly small apparent 
dielectric response $\epsilon_{i} \simeq 2$ independently of the slab thickness. First, while the model critically depends on this assumption, it could not be directly confirmed because specific probing of the interfacial permittivity remains a challenge for experiments; further, as detailed below, local dielectric constant calculations from molecular dynamics simulations with slab geometry boundary conditions are notoriously difficult to converge. ${ }^{5,7,23,25}$ A second difficulty for this postulate is that it would require an extreme alignment of interfacial water dipoles, a situation in stark contrast with the moderate structural perturbation of water at graphene interfaces found in a series of recent molecular dynamics simulations. ${ }^{26-29}$ Also, it has been proposed ${ }^{16}$ that the slower reorientation dynamics of water at the interface compared to the bulk would be consistent with the very low dielectric constant. However, the static dielectric constant is not a dynamical property, and in any event our recent work has shown ${ }^{26,27}$ that interfacial water dynamics is too moderately affected to be consistent with any extreme restriction of molecular orientations. ${ }^{30}$

To address the dielectric constant issue, we will use molecular dynamics simulations of water confined between graphene plates with different separations. We show that the dielectric constant reduction does not arise from a surface-induced alignment of water molecules; instead it is due to the dipolar electric field anisotropy and the replacement of water molecules with a nonpolarizable wall in a region where dipoles are positively correlated. This two-fold source explains why simulations found the dielectric constant reduction to be common ${ }^{6,23}$ to all confined polar solvents. The two-state description involving frozen interfacial and bulklike populations is shown to be inadequate. This failure results, first, from the decay of the walls' effect over several nanometers inside the slab due to the large dipole-dipole correlation length, and second, from the interfacial properties' dependence on the wall separation.

In our study, we performed classical molecular dynamics simulations of a slab of SPC/E water molecules confined between two rigid single graphene sheets and periodically replicated in the two directions parallel to the graphene planes. Increasing sheet separations of 15, 50, 80 and $110 \AA$ were considered. The Yeh-Berkowitz ${ }^{8}$ slab correction to Ewald summation 
was employed. A $80 \times 80 \times 80 \AA^{3}$ neat water system was simulated for reference. For each system, trajectories between 150 ns and $4.5 \mu$ s were propagated with Gromacs $2021^{31}$ at 300 K. Details are provided in SI. All reported error bars are $95 \%$ confidence intervals.

First, we determine the local dielectric constant changes across the sample. The static dielectric constant is defined by the system's polarization response to an electric field, and the classic Kirkwood-Fröhlich ${ }^{32}$ theory has established that the constant is related to the sample's polarization fluctuations. Prior studies ${ }^{25,33}$ have shown that under the assumptions of local and linear polarization response to the field, a local dielectric constant can be defined, related to the local polarization density and total dipole correlation. For a two-dimensional liquid slab periodic along $x$ and $y$, and under constant electric displacement along $z$, the local dielectric constant in $z$ is ${ }^{25,33}$

$$
\frac{1}{\epsilon_{\perp}(z)}=1-4 \pi \beta\left[\left\langle m_{\perp}(z) M_{\perp}\right\rangle-\left\langle m_{\perp}(z)\right\rangle\left\langle M_{\perp}\right\rangle\right]
$$

where $m_{\perp}(z)$ is the local polarization density along the $z$ axis, averaged in the plane parallel to the graphene wall, $M_{\perp}$ is the total dipole and $\beta=1 / k_{B} T$. Following ref. $5, m_{\perp}(z)$ is defined from the water point charge density $\rho(z)$ as $m_{\perp}(z)=-\int_{0}^{z} \rho\left(z^{\prime}\right) \mathrm{d} z^{\prime}$, where the simulation box extends between 0 and $L_{z}$ along the $z$ direction. Although eq. 1 can formally be used to determine a local $\epsilon_{\perp}^{-1}(z)$ value with an arbitrary spatial resolution, the underlying local response approximation will break down for resolutions finer than the molecular dimension, ${ }^{25}$ and a physically meaningful effective dielectric constant requires an average over one (or more) molecular layers.

The $\epsilon_{\perp}^{-1}(z)$ profile calculated for a $50 \AA$ wall separation is reported in Fig. 1. In agreement with recent calculations on similar systems, ${ }^{6,7,23}$ our results show that the local dielectric constant is strongly affected by the walls over more than two molecular layers, and approaches the bulk value in the middle of the slab. However, while these dielectric constant profiles correctly describe the polarization response to an external applied field, ${ }^{6,34}$ their determination 
and interpretation remain complex. First, obtaining a precise estimate of $\epsilon_{\perp}(z)$ is challenging due to the difficulty in converging these profiles even with considerable simulation lengths (here $4.5 \mu \mathrm{s}$ ). Second, the profile exhibits rapid and large amplitude fluctuations, at times reaching negative $\epsilon_{\perp}^{-1}(z)$ values (suggested to reflect an overscreening of the field ${ }^{23,35,36}$ and also present in the bulk wavevector-dependent dielectric function ${ }^{35}$ ), so that averages over molecular layers should be considered. Simplified models with box profiles for the dielectric constant have been developed to reproduce the average dielectric constant over some regions, ${ }^{23}$ but the molecular factors causing the wall's dramatic reduction of the water dielectric constant still remain to be elucidated.

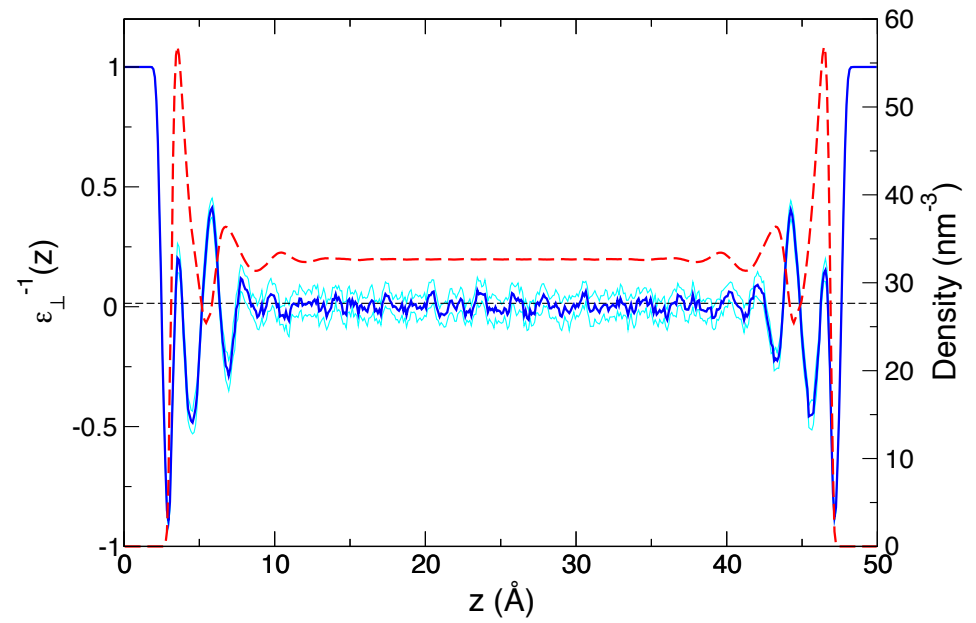

Figure 1: Inverse local dielectric constant profile $\epsilon_{\perp}^{-1}(z)$ across the water slab eq. 1 (blue solid lines, with confidence interval in cyan), bulk $\epsilon_{b}^{-1} \simeq 1 / 71$ reference (black dashes) and molecular number density profile (red dashes).

A precise molecular connection between dielectric constant and correlations between dipoles is provided by the Kirkwood factor, and dipolar correlations have been extensively studied for dipolar liquids. ${ }^{37-43}$ Inspired by recent work ${ }^{42-44}$ on the calculation of neat water's dielectric constant from the Kirkwood factor, we have borrowed some ideas from these efforts to obtain a qualitative molecular understanding of the confined water dielectric constant.

Before addressing the complex confined case, we consider the reference situation of neat 
liquid water without confinement. For a homogeneous isotropic liquid, the $r$-dependent Kirkwood function defined as ${ }^{40,41,43}$

$$
G_{K}(r)=\left\langle\boldsymbol{\mu}^{(i)} \cdot \mathbf{M}^{(i)}(r)\right\rangle / \mu^{2}
$$

probes the correlation between the dipole $\boldsymbol{\mu}^{(i)}$ of molecule $i$ and the total dipole $\mathbf{M}^{(i)}(r)$ due to all molecules within a sphere of radius $r$ centered on molecule $i$. Within an infinite system, $G_{K}(r)$ reaches a plateau value $g_{K}$ for $r$ larger than the Kirkwood correlation length $r_{K}$,

$$
g_{K}=G_{K}\left(r>r_{K}\right) .
$$

$g_{K}$ is the Kirkwood g-factor and the dielectric constant is determined by both the dipole density and an orientational correlation contribution probed by $g_{K},{ }^{32}$

$$
4 \pi \beta \rho_{N} \mu^{2} g_{K}=\frac{(\epsilon-1)(2 \epsilon+1)}{\epsilon},
$$

where $\rho_{N}$ is the dipole number density and $\mu$ is the molecular dipole amplitude.

However, the situation is rendered more complex by the periodic boundary conditions used in molecular dynamics simulations (even for large systems): the electrostatic boundary conditions used in the Ewald summation critically affect the relation between dielectric constant and dipole fluctuations. ${ }^{38}$ The typical tin-foil conditions correspond to embedding the range of periodic system replicas in a $\epsilon^{\prime}=\infty$ continuum (zero macroscopic field) and preferentially stabilize large fluctuations of the system total dipole. In contrast, embedding the system in vacuum $\left(\epsilon^{\prime}=0\right.$, i.e. zero electric displacement) tends to depolarize the system. Electrostatic boundary conditions thus cause long-range dipole correlations that add to the correlations present in an infinite system. The Kirkwood function obtained from periodic boundary condition simulations is the sum of the infinite system Kirkwood function and of these long-range correlations, and may no longer exhibit the large- $r$ plateau that defines the 
Kirkwood g-factor in infinite systems. This therefore precludes the simple application of the Kirkwood expression eq. 4 to obtain the dielectric constant.

Recent work on neat water ${ }^{42-44}$ has proposed an elegant approach to cancel these longrange terms - and thus provide a much closer approach to the infinite bulk liquid description - by combining the Kirkwood functions obtained with $\epsilon^{\prime}=\infty$ and $\epsilon^{\prime}=0$ conditions. Based on a prior study ${ }^{40}$ which had established the analytic expression for these long-range corrections in a homogeneous dipolar liquid, the Kirkwood function corrected for periodic boundary condition effects was determined ${ }^{42}$ to be $G_{K c}(r)=\left(2 G_{K}^{\epsilon^{\prime}=\infty}(r)+G_{K}^{\epsilon^{\prime}=0}(r)\right) / 3$. We have performed molecular dynamics simulations for a large $80 \times 80 \times 80 \AA^{3}$ box of neat water respectively embedded in $\epsilon^{\prime}=\infty$ and $\epsilon^{\prime}=0$ dielectric media and determined the corrected Kirkwood function $G_{K c}(r)$. In agreement with ref. 42, Fig. 2a shows that $G_{K c}(r)$ exhibits a plateau after a few molecular layers at approximately $r=10 \AA$.

However, as we now show, dipoles remain correlated much beyond this distance. To this end, rather than perform the usual average of the Kirkwood function over all orientations around the central dipole $i$, we decompose the total dipole $\mathbf{M}^{(i)}(r)$ due to all molecules within a sphere of radius $r$ around molecule $i$ into its contributions $\mathbf{M}^{(i)}(r, \theta)$ arising from those molecules $j$ such that the $\mathbf{r}_{i j}=\mathbf{r}_{j}-\mathbf{r}_{i}$ vector is at an angle $\theta$ with the $\boldsymbol{\mu}^{(i)}$ dipole vector, and define the angle-dependent Kirkwood function

$$
G_{K}(r, \theta)=\left\langle\boldsymbol{\mu}^{(i)} \cdot \mathbf{M}^{(i)}(r, \theta)\right\rangle / \mu^{2}
$$

such that $G_{K}(r)=\int_{0}^{\pi} \mathrm{d} \theta \sin \theta G_{K}(r, \theta)$. Figure $2 \mathrm{~b}$ shows that in the direction of the $\boldsymbol{\mu}^{(i)}$ dipole, the dipole correlation probed by $G_{K}(r, \theta)$ has not reached a constant value by $40 \AA$. This behavior may be understood by noting that the dipole-dipole interaction scales as $\left(3 \cos ^{2}(\theta)-1\right) / r^{3}$ (see SI), and that its angular average vanishes; thus, after a few molecular distances in an isotropic homogeneous dipolar liquid - i.e. once the intermolecular correlations are well described by the point dipole potential $-G_{K}(r)$ reaches a constant value, 
despite the continuing existence of ongoing angular correlations. These results show that the correlation between a molecular dipole and the total surrounding dipole is both strongly anisotropic and decays on a distance of several nanometers. Both these points will be very important for the anisotropic slab conditions that we now address.
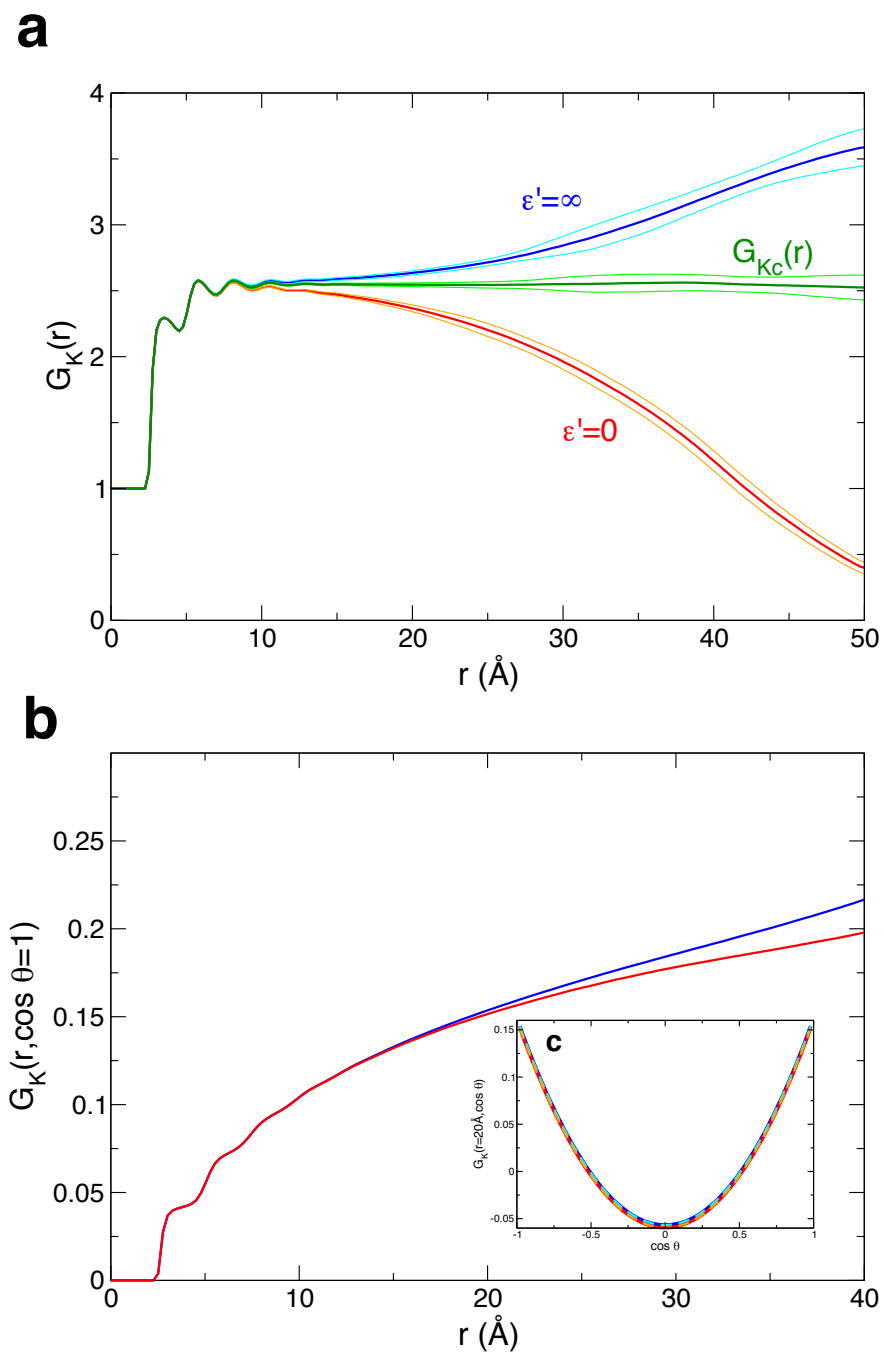

Figure 2: a) Kirkwood function $G_{K}(r)$ eq. 2 in neat water with $\epsilon^{\prime}=\infty$ (blue) and $\epsilon^{\prime}=0$ (red) boundary conditions and the resulting corrected Kirkwood function $G_{K c}(r)=\left(2 G_{K}^{\epsilon^{\prime}=\infty}(r)+\right.$ $\left.G_{K}^{\epsilon^{\prime}=0}(r)\right) / 3$ (green), together with the confidence intervals. b) Angularly resolved Kirkwood function eq. $5 G_{K}(r, \theta=0)$ from $\epsilon^{\prime}=\infty$ (blue) and $\epsilon^{\prime}=0$ (red) simulations c) $G_{K}(r=$ $20 \AA, \theta)$ from $\epsilon^{\prime}=\infty$ (blue) and $\epsilon^{\prime}=0$ (red) simulations together with the second Legendre polynomial fit $\left(3 \cos ^{2} \theta-1\right) / 2$ (dashes) (the $\perp$ notation used in the slab situation is dropped here since neat water is isotropic).

To analyze the local dielectric constant of confined water along the direction normal to 
the walls, we start by extending the $r$-dependent Kirkwood function eq. 2 to describe the dependence of dipolar correlations on both the distance $r$ and the location $z$ across the slab,

$$
G_{K}^{\perp}(r ; z)=\frac{1}{\left\langle\left(\mu_{\perp}^{(i)}\right)^{2}\right\rangle_{z_{i}=z}}\left[\left\langle\mu_{\perp}^{(i)} M_{\perp}^{(i)}(r)\right\rangle_{z_{i}=z}-\left\langle\mu_{\perp}^{(i)}\right\rangle_{z_{i}=z}\left\langle M_{\perp}^{(i)}(r)\right\rangle_{z_{i}=z}\right]
$$

$G_{K}^{\perp}(r ; z)$ probes the correlation between the perpendicular component of the dipole of molecule $i$ lying at a position $z_{i}=z$ and the perpendicular component of the total dipole $M_{\perp}^{(i)}(r)$ due to all molecules within a sphere of radius $r$ centered on molecule $i{ }^{45}$ The associated $r$-and $z$-dependent local dielectric constant is defined by the extension of eq. 4

$$
12 \pi \beta \rho_{N}(z)\left\langle\mu_{\perp}^{2}(z)\right\rangle G_{K c}^{\perp}(r ; z)=\frac{\left(\epsilon_{\perp}(r ; z)-1\right)\left(2 \epsilon_{\perp}(r ; z)+1\right)}{\epsilon_{\perp}(r ; z)}
$$

where $G_{K c}^{\perp}(r ; z)$ is the Kirkwood function eq. 6 corrected for the electrostatic boundary conditions used in the simulations.

However, the correction of this new Kirkwood function eq. 6 for the electrostatic boundary conditions is more complex than in the homogeneous solvent eq. 2. The inhomogeneous character of the confined water slab implies that the theoretical developments applied to a homogeneous isotropic dipolar liquid ${ }^{40}$ and the procedure designed ${ }^{42}$ to correct the Kirkwood function for electrostatic boundary conditions and described above for neat water are no longer directly applicable. A formal extension of the analytic approach of ref. 40 to an anisotropic system would be desirable, but appears to be quite challenging. Here we will adopt a simple approximate numerical method which will be shown to prove useful.

Our slab simulations are performed with the Yeh-Berkowitz ${ }^{8}$ slab correction to the threedimensional Ewald summation, which has been shown ${ }^{44}$ to be equivalent to zero electric displacement conditions $(\boldsymbol{D}=0)$ along the direction normal to the slab. These conditions are expected to depolarize the system and add a negative contribution to the Kirkwood function. This contribution needs to be compensated in order to obtain the Kirkwood func- 
tion corresponding to an infinite system that is required in eq. 7 to determine the dielectric constant. We first note that in the homogeneous liquid, the electrostatic boundary condition correction scales ${ }^{40}$ as the ratio between the volume $v(r)$ of sphere of radius $r$ 's portion that remains within the simulation box and the simulation box's total volume $V=L_{x} \times L_{y} \times L_{z}$, with an amplitude that depends on the dielectric constants of the system and of the embedding medium. We will therefore perform simulations of very large slab boxes, so that the Kirkwood function eq. 6 can reach an approximately constant value before the $v(r) / V$ term (and the correction) becomes too important. In addition, we estimate the necessary electrostatic boundary condition correction by assuming that it approximately scales as $v(r) / V$ in the slab, and determine its amplitude $a$ from a numerical fit $(a v(r) / V+b)$ at very large $r$ distances, following what had been done in ref. 41 for the neat water case. We therefore approximate the corrected Kirkwood function as

$$
G_{K c}^{\perp}(r ; z) \simeq G_{K}^{\perp}(r ; z)-a v(r) / V
$$

As shown in the SI, this procedure provides a very good description of the large- $r$ behavior of the Kirkwood function eq. 6 in the middle of the slab where the liquid is homogeneous and where the correction amplitude $a$ is large; this approach is more approximate closer to the wall, but there the magnitude of the correction is smaller. Finally, we verify that the shortrange part of the Kirkwood function that we analyze does not significantly change when the simulation box size is changed (see SI). Certainly, this approach is only semi-quantitative, but as we now show, it offers a useful way to achieve our central goal of gaining a microscopic understanding of the changes in the water dielectric constant due to confinement.

Figures $3 \mathrm{a}$ and b show first the $G_{K}^{\perp}(r ; z)$ and $G_{K c}^{\perp}(r ; z)$ profiles around water molecules at increasing $z$ distances from the graphene wall and then the associated $r$-dependent dielectric constants $\epsilon_{\perp}(r ; z)$ from eq. 7 . We first examine the profile in the middle of the $110 \AA$-thick box, i.e. at a distance of $>45 \AA$ from the walls. The short-range part of $G_{K}^{\perp}(r ; z)$ there is 
very similar to that found in neat water (Fig. 2a), while its long-range part exhibits a decay with $r$ due to depolarization induced by the electrostatic boundary conditions that scales as $r^{3}$ (see SI). Compensating for the latter via eq. 8 yields a $G_{K c}^{\perp}(r ; z)$ corrected Kirkwood function that is constant beyond $\simeq 10 \AA$, and the resulting long-range limit of the $r$-dependent dielectric constant eq. 7 yields a value of $\epsilon_{\perp}(z) \simeq 71.5$; this shows that the bulk dielectric constant is recovered at very large distances from the walls.

We now contrast this with the behavior in the first layer next to the wall $(2.5<z<5.0 \AA)$. The corrected Kirkwood function profile (Fig. 3b) is strongly reduced with respect to that in the middle of the slab just discussed, which leads to a dramatic drop in the $r$-dependent dielectric constant profile (Fig. 3b). To understand this first layer behavior, we need to appreciate that both the corrected Kirkwood function and the local dielectric constant result from a sum of dipole correlations over three main $r$-separation ranges; we examine the contributions of these successive terms to the overall change $\Delta \epsilon$ in the dielectric constant.

The first contribution comes from dipole self correlations: $\epsilon_{\perp}(r=0 ; z)$ is determined by the $\left\langle\mu_{\perp}^{2}(z)\right\rangle$ autocorrelation of molecular dipole $z$ components. While the water molecules' orientations are distributed isotropically in the neat liquid and in the middle of the slab, molecules at the interface tend to lie with their $\mathrm{OH}$ groups tangent to the wall. ${ }^{27}$ This leads to a decrease in $\left\langle\mu_{\perp}^{2}\right\rangle^{6,46}$ from $1.84 \mathrm{D}^{2}$ to $1.09 \mathrm{D}^{2}$, which causes a dielectric constant reduction of just $\Delta \epsilon \simeq-10$. This result thus shows that the usual explanation of confined water's very low dielectric constant by the orientational restrictions imposed by the wall on the interfacial molecules only accounts for a small fraction of the overall dielectric constant reduction.

The largest contribution actually comes from an effect on the two other terms arising from intermolecular correlations, now discussed. Short-ranged intermolecular correlations arise from molecules in the first or second shell of each other $(2.5<r<8.5 \AA)$, where the fluctuations in $G_{K c}^{\perp}(r ; z)$ and $\epsilon_{\perp}(r ; z)$ reflect the liquid radial density profile: the decrease between the middle of the slab and the interfacial layer results from the reduced number of neighbors around a molecule next to the wall. ${ }^{47}$ Long-ranged intermolecular correlations 

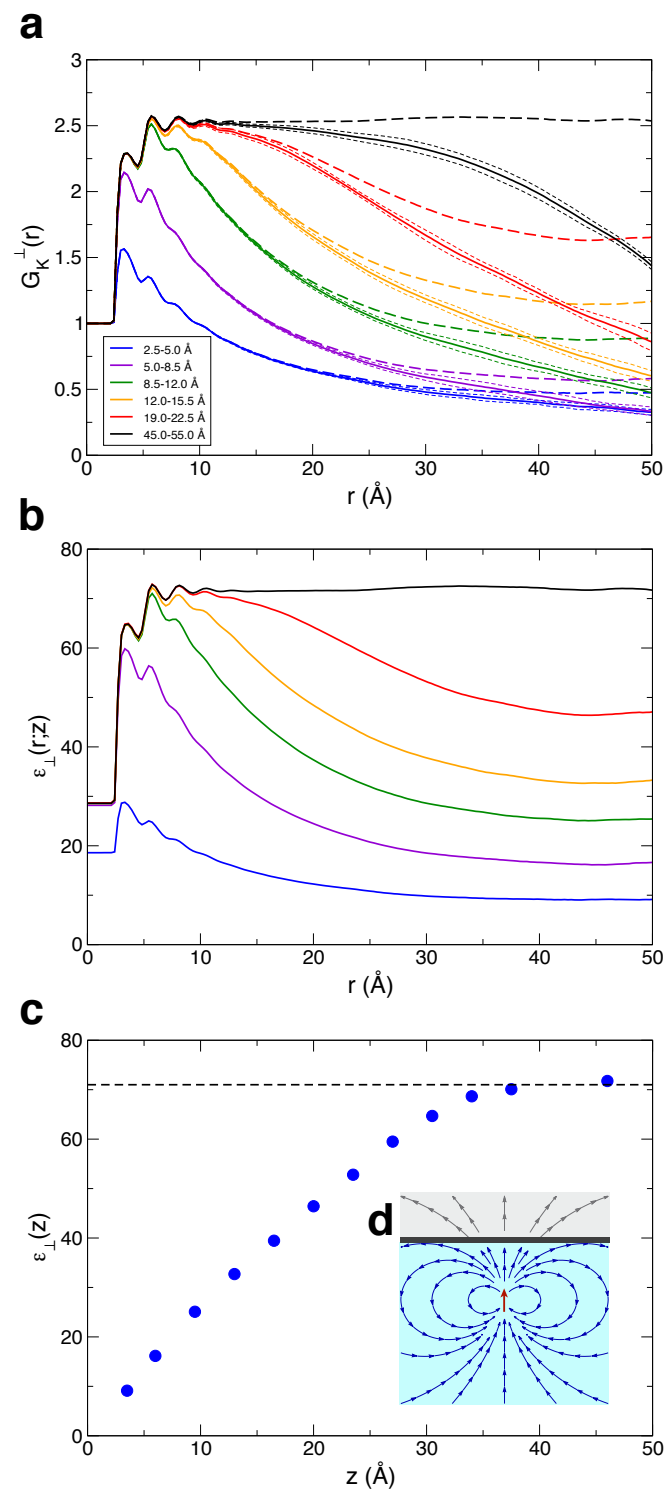

Figure 3: a) Kirkwood function $G_{K}^{\perp}(r ; z)$ eq. 6 at a dipole-dipole separation of $r$ around a molecule at a series of distances $z$ from the graphene wall (solid lines), calculated from a large simulation box of $110.68 \times 110.76 \times 110 \AA^{3}$ (see SI). Also displayed are their confidence intervals (short dashes) and the corrected Kirkwood function $G_{K c}^{\perp}(r ; z)$ eq. 8 (long dashes); b) Separation-dependent local dielectric constant $\epsilon_{\perp}(r ; z)$ eq. 7 for the same situations as in a); c) Local dielectric constant profile $\epsilon_{\perp}(z)$ determined from the $\epsilon_{\perp}(r ; z)$ profiles at $r=40 \AA$, together with the bulk reference $\epsilon_{b}$ value (dashes); d) schematic representation of the dipolar electric field produced by a dipole perpendicular to the wall and showing the excluded volume effect.

are due to dipole-dipole correlations: the intermolecular dipole correlations' decrease at the interface with respect to the slab's middle is shown by Fig. 3 b to cause a very large $\Delta \epsilon \simeq-52$ 
reduction in the dielectric constant. A two-dimensional decomposition of $G_{K}^{\perp}(r ; z)$ along the distance $r$ between the dipoles and the orientation of their separation vector relative to the wall normal direction is instructive here (see Fig. S7 in SI). It shows that the decreased correlation is essentially due to the volume excluded by the wall rather than to a change in the intermolecular dipoles' correlation between the confined water molecules compared to the neat liquid. As shown in Fig. 2b for neat water, the dipolar field is strongly anisotropic and water dipoles remain correlated over several nanometers. In the bulk, water molecules that lie around a central dipole within a cone - whose axis is aligned with this dipole and whose semiangle is the magic angle - are positively correlated with the central dipole. In the slab geometry, as illustrated in Fig. 3d, the wall's presence induces a shape factor by selectively excluding water molecules in the direction of the $\mu_{\perp}$ vector component that, in the bulk, gave a positive correlation. Thus, this exclusion reduces the angle-averaged dipoledipole correlation that enters in the Kirkwood factor. As shown in the SI, this wall excluded volume effect on the average dipole correlation can be determined analytically for a dipolar fluid at a distance $z$ from the wall and its $r$-dependence yields a good description of the $G_{K c}^{\perp}(r ; z) r$-decay obtained from our simulations in Fig. 3a over the 10-40 $\AA$ range.

The analysis that was just described for the first layer is then repeated for a series of molecular layers at increasing distances $z$ from the wall. Figures $3 \mathrm{a}$ and $\mathrm{b}$ show that the dipole correlations remain affected by the presence of the wall up to distances of several nanometers, a feature consistent with the long-range dipole correlation determined in neat water in absence of confinement. For example, at $19.0<z<22.5 \AA, G_{K c}^{\perp}(r ; z)$ exhibits the same behavior as that in the bulk up to $r$ distances of $\simeq 12 \AA$, when the sphere around the central dipole approaches the first two layers next to the wall where the dipole orientations are perturbed by the wall; for $r>12 \AA$ distances, $G_{K c}^{\perp}(r ; z)$ markedly drops, reflecting the volume excluded by the wall.

Figure 3c reports the local dielectric constant $\epsilon_{\perp}(z)$ determined from the long-range plateau value of $\epsilon_{\perp}(r ; z)$ in Fig. 3b. While the values should be considered as approximate 
estimates, they show two important features: that the dielectric constant is strongly reduced at the interface $\left(\epsilon_{\perp} \simeq 9\right)$, and that the bulk value is gradually recovered only over a distance of several nanometers.

These results show that the typical capacitor model - based on a combination of bulk-like and ice-like populations - often used to interpret the average dielectric constant of confined water does not correctly describe the confined liquid water situation. First, a description involving only two populations with extreme dielectric constants of $\epsilon_{i}=2$ and $\epsilon_{b}=71$ is an oversimplification: the dielectric constant reduction is not due to a local structural restriction induced by the wall but instead is caused by a long-range dipolar correlation effect; the local dielectric constant gradually approaches the bulk value over distances of many molecular layers, and multiple populations with distinct dielectric constants would need to be accounted for. Beyond that necessary accounting, the dielectric properties at the interface and in the middle of the slab are not independent of the slab thickness, in contrast to the capacitor model's underlying assumption. Figure 4 demonstrates this by comparing the Kirkwood functions in the first two layers next to the wall when the interwall distance is changed from $110 \AA$ to $15 \AA$. Due to the long-range dipole correlation, at the smaller separation, the interfacial layer is affected by both walls and the Kirkwood function is further reduced when there are two walls instead of one. All of the features discussed in this paragraph show that the dielectric constant of $\simeq 2$ determined experimentally ${ }^{10}$ for an extremely thin slab with a single layer of water cannot be taken to be representative of the dielectric constant at the interface for larger slab thickness.

We finally quickly comment on the confinement's effect on the water dielectric constant along the direction parallel to the wall. As found in recent simulations, ${ }^{46}$ our results show that this component of the dielectric tensor is strongly enhanced with respect to the bulk dielectric constant, but that this effect does not extend further than a few molecular layers from the wall (see SI). In agreement with the suggestion of ref. 46, we find that this enhancement arises mostly from the orientation of interfacial water molecules which, typically lying 


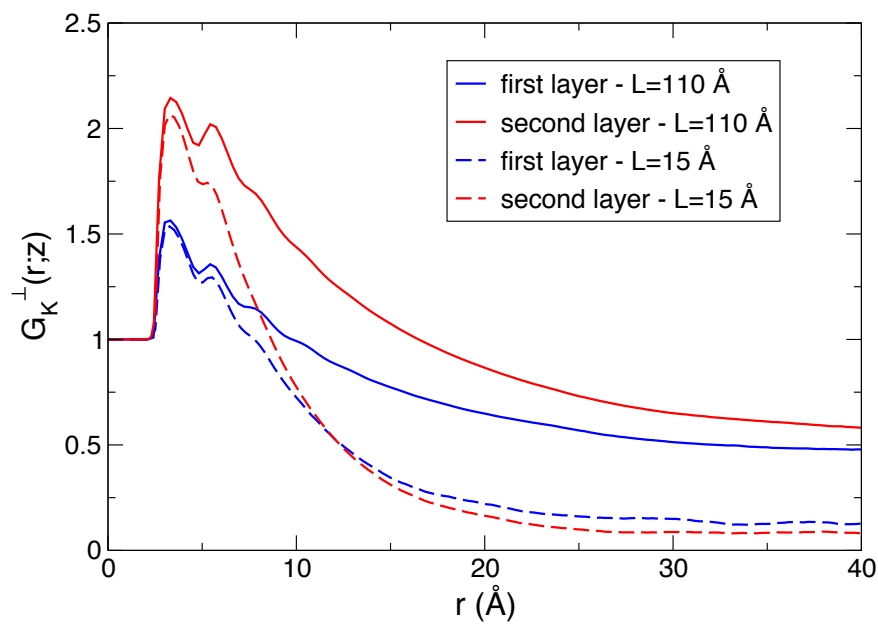

Figure 4: Corrected Kirkwood function $G_{K c}^{\perp}(r ; z)$ eq. 8 determined in the first (blue) and second (red) water layers next to the wall for a distance between the walls of $110 \AA$ (solid lines) and $15 \AA$ (dashes).

tangent to the wall, increase the $\left\langle\mu_{\|}^{2}\right\rangle$ average dipole projection. This structural origin therefore explains why the $\epsilon_{\|}(z)$ enhancement only occurs close to the wall and why it sensitively depends on the wall's nature. ${ }^{19}$ The long-range dipolar field shape factor responsible for the perpendicular component's reduction further enhances the parallel dielectric constant, but this effect is much weaker in this geometry than in the perpendicular situation (see SI).

In conclusion, our study has shown that the low perpendicular dielectric constant that has been measured for water confined within nanometer-sized channels is not due to aligned 'ice-like' water molecules at the interface. This reduction instead arises from the anisotropy of the dipolar electric field and from the low dielectric constant of the surrounding medium; this produces an excluded-volume effect in which water molecules are excluded from a region where, in the bulk, they bring a positive dipole correlation. Due to the long-range character of the dipole-dipole interaction, the impact of the wall on the local dielectric constant extends over several nanometers within the slab, contrasting with the traditional capacitor series model assumptions. Our results thus show that the large reduction in dielectric constant across the confined water slab is mostly a consequence of the low-dielectric envi- 
ronment in which it is embedded, and not of specific interfacial water properties. This is consistent with the low dielectric constant of the surrounding media in all observations of confined water dielectric constant reduction so far - e.g. boron nitride $(\epsilon \simeq 3)$ channels in the experiments ${ }^{10}$ and apolar walls surrounded by vacuum in simulations ${ }^{6,8,9,21,46}$ - and with the universal character of the dielectric constant reduction observed for a series of confined polar solvents. ${ }^{6}$ One important consequence of this environmental impact is that changing the confining material dielectric properties (e.g. replacing graphene with metallic walls) could be used to control the confined water dielectric constant. In a broader view, these results have very important implications, e.g. for mesoscopic continuum models which require the water dielectric constant as an input, for water and ion transport inside artificial and biological nanochannels ${ }^{11,12,48,49}$ and mesoporous material cavities ${ }^{50}$ and for chemical reactivity in aqueous nanodroplets ${ }^{51,52}$ and at electrodes. ${ }^{13}$

\section{Acknowledgement}

D.L. thanks Prof. M. Sprik (Univ. Cambridge) for his valuable comments and suggestions. This work has been supported through a CDSN PhD fellowship to J.F.O. from the French ministry of higher education and research.

\section{Supporting Information Available}

The following files are available free of charge. Simulation details, parallel dielectric constant results, angularly-resolved Kirkwood function, derivation of dipole-dipole correlation next to a wall. 


\section{References}

(1) Levinger, N. E. Water in confinement. Science 2002, 298, 1722-1723.

(2) Rasaiah, J. C.; Garde, S.; Hummer, G. Water in nonpolar confinement: from nanotubes to proteins and beyond. Annu Rev Phys Chem 2008, 59, 713-740.

(3) Zhang, C.; Gygi, F.; Galli, G. Strongly anisotropic dielectric relaxation of water at the nanoscale. J Phys Chem Lett 2013, 4, 2477-2481.

(4) De Luca, S.; Kannam, S.; Todd, B.; Frascoli, F.; Hansen, J.; Daivis, P. Effects of Confinement on the Dielectric Response of Water Extends up to Mesoscale Dimensions. Langmuir 2016, 32, 4765-4773.

(5) Schlaich, A.; Knapp, E. W.; Netz, R. R. Water Dielectric Effects in Planar Confinement. Phys Rev Lett 2016, 117, 048001.

(6) Motevaselian, M. H.; Aluru, N. R. Universal Reduction in Dielectric Response of Confined Fluids. ACS Nano 2020, 14, 12761-12770.

(7) Ruiz-Barragan, S.; Muñoz-Santiburcio, D.; Körning, S.; Marx, D. Quantifying anisotropic dielectric response properties of nanoconfined water within graphene slit pores. Phys Chem Chem Phys 2020, 22, 10833-10837.

(8) Yeh, I.-C.; Berkowitz, M. L. Ewald summation for systems with slab geometry. J Chem Phys 1999, 111, 3155-3162.

(9) Senapati, S.; Chandra, A. Dielectric constant of water confined in a nanocavity. J Phys Chem B 2001, 105, 5106-5109.

(10) Fumagalli, L.; Esfandiar, A.; Fabregas, R.; Hu, S.; Ares, P.; Janardanan, A.; Yang, Q.; Radha, B.; Taniguchi, T.; Watanabe, K. et al. Anomalously low dielectric constant of confined water. Science 2018, 360, 1339-1342. 
(11) Striolo, A.; Michaelides, A.; Joly, L. The Carbon-Water Interface: Modeling Challenges and Opportunities for the Water-Energy Nexus. Annu Rev Chem Biomol Eng 2016, 7, 533-556.

(12) Lynch, C.; Rao, S.; Sansom, M. Water in Nanopores and Biological Channels: A Molecular Simulation Perspective. Chem Rev 2020, 120, 10298-10335.

(13) Bangle, R.; Schneider, J.; Conroy, D.; Aramburu-Trošelj, B.; Meyer, G. Kinetic Evidence That the Solvent Barrier for Electron Transfer Is Absent in the Electric Double Layer. J Am Chem Soc 2020, 142, 14940-14946.

(14) Loche, P.; Ayaz, C.; Schlaich, A.; Bonthuis, D.; Netz, R. Breakdown of Linear Dielectric Theory for the Interaction between Hydrated Ions and Graphene. J Phys Chem Lett 2018, 9, 6463-6468.

(15) Zhang, C. Note: On the dielectric constant of nanoconfined water. J Chem Phys 2018, $148,156101$.

(16) Varghese, S.; Kannam, S.; Hansen, J.; P Sathian, S. Effect of Hydrogen Bonds on the Dielectric Properties of Interfacial Water. Langmuir 2019, 35, 8159-8166.

(17) Zhu, H.; Yang, F.; Zhu, Y.; Li, A.; He, W.; Huang, J.; Li, G. Investigation of dielectric constants of water in a nano-confined pore. RSC Advances 2020, 10, 8628-8635.

(18) Deißenbeck, F.; Freysoldt, C.; Todorova, M.; Neugebauer, J.; Wippermann, S. Dielectric properties of nano-confined water: a canonical thermopotentiostat approach. Phys Rev Lett 2021, 126, 136803.

(19) Qi, C.; Zhu, Z.; Wang, C.; Zheng, Y. Anomalously Low Dielectric Constant of Ordered Interfacial Water. J Phys Chem Lett 2021, 12, 931-937.

(20) Mondal, S.; Bagchi, B. Water Layer at Hydrophobic Surface: Electrically Dead but Dynamically Alive. Nano Letters 2020, 20, 8959-8964. 
(21) Mondal, S.; Bagchi, B. Anomalous dielectric response of nanoconfined water. J Chem Phys 2021, 154, 044501.

(22) Stengel, M.; Spaldin, N. Origin of the dielectric dead layer in nanoscale capacitors. Nature 2006, 443, 679-682.

(23) Loche, P.; Ayaz, C.; Wolde-Kidan, A.; Schlaich, A.; Netz, R. R. Universal and NonUniversal Aspects of Electrostatics in Aqueous Nano-Confinement. J Phys Chem B 2020, 124, 4365-4371.

(24) Jalali, H.; Ghorbanfekr, H.; Hamid, I.; Neek-Amal, M.; Rashidi, R.; Peeters, F. Outof-plane permittivity of confined water. Phys Rev E 2020, 102, 022803.

(25) Ballenegger, V.; Hansen, J. Dielectric permittivity profiles of confined polar fluids. $J$ Chem Phys 2005, 122, 114711.

(26) Zhang, Y.; Stirnemann, G.; Hynes, J. T.; Laage, D. Water dynamics at electrified graphene interfaces: a jump model perspective. Phys Chem Chem Phys 2020, 22, $10581-10591$.

(27) Zhang, Y.; de Aguiar, H. B.; Hynes, J. T.; Laage, D. Water Structure, Dynamics, and Sum-Frequency Generation Spectra at Electrified Graphene Interfaces. J Phys Chem Lett 2020, 11, 624-631.

(28) Cicero, G.; Grossman, J.; Schwegler, E.; Gygi, F.; Galli, G. Water confined in nanotubes and between graphene sheets: a first principle study. J Am Chem Soc 2008, 130, $1871-1878$.

(29) Ho, T. A.; Striolo, A. Molecular dynamics simulation of the graphene--water interface: comparing water models. Mol Simulat 2014, 40, 1190-1200.

(30) Ref. 20 suggested that interfacial water dynamics, although not very different from that in further layers, is affected by the suppression of jump hydrogen-bond exchanges and a 
caging effect, leading to dielectric constant reduction; ${ }^{21}$ however, our prior work ${ }^{26,27}$ had showed that water jump hydrogen-bond exchange dynamics are only very moderately affected by a graphene interface.

(31) Abraham, M. J.; Murtola, T.; Schulz, R.; Páll, S.; Smith, J. C.; Hess, B.; Lindahl, E. GROMACS: High performance molecular simulations through multi-level parallelism from laptops to supercomputers. SoftwareX 2015, 1, 19-25.

(32) Kirkwood, J. G. The Dielectric Polarization of Polar Liquids. J Chem Phys 1939, 7, $911-919$.

(33) Stern, H. A.; Feller, S. E. Calculation of the dielectric permittivity profile for a nonuniform system: Application to a lipid bilayer simulation. J Chem Phys 2003, 118, 3401-3412.

(34) Loche, P.; Wolde-Kidan, A.; Schlaich, A.; Bonthuis, D.; Netz, R. Comment on "Hydrophobic Surface Enhances Electrostatic Interaction in Water". Phys Rev Lett 2019, 123, 049601.

(35) Bopp, P. A.; A, K. A.; G, S. Static Nonlocal Dielectric Function of Liquid Water. Phys. Rev. Lett. 1996, 76, 1280-1283.

(36) Bonthuis, D. J.; Gekle, S.; Netz, R. R. Profile of the static permittivity tensor of water at interfaces: consequences for capacitance, hydration interaction and ion adsorption. Langmuir 2012, 28, 7679-7694.

(37) Deutch, J. M. Statistical Mechanics of Simple Polar Fluids. Annu Rev Phys Chem 1973, 24, 301-323.

(38) de Leeuw, S. W.; Perram, J. W.; Smith, E. R. Simulation of electrostatic systems in periodic boundary conditions. I. Lattice sums and dielectric constants. Proc $R$ Soc Lond A 1980, 373, 27-56. 
(39) Madden, P.; Kivelson, D. A consistent molecular treatment of dielectric phenomena. Adv Chem Phys 1984, 56, 467-566.

(40) Caillol, J. Asymptotic behavior of the pair-correlation function of a polar liquid. J Chem Phys 1992, 96, 7039-7053.

(41) Fulton, R. The effect of the spatial nonlocality of the Kirkwood g-factor on the determination of the long wavelength dielectric functions in dipolar fluids. $J$ Chem Phys 2012, 136, 084502.

(42) Zhang, C.; Sprik, M. Computing the dielectric constant of liquid water at constant dielectric displacement. Phys Rev B 2016, 93, 144201.

(43) Zhang, C.; Hutter, J.; Sprik, M. Computing the Kirkwood G-factor by combining constant Maxwell electric field and electric displacement simulations: Application to the dielectric constant of liquid water. J Phys Chem Lett 2016, 7, 2696-2701.

(44) Zhang, C.; Sayer, T.; Hutter, J.; Sprik, M. Modelling electrochemical systems with finite field molecular dynamics. J. Phys. Energy 2020, 2, 032005.

(45) We stress the difference between our definition of the total dipole entering in the Kirkwood factor and prior studies in slab geometries which had either considered the dipole within a very small sphere ${ }^{53}$ or within the entire system. ${ }^{54}$

(46) Motevaselian, M.; Aluru, N. Confinement-Induced Enhancement of Parallel Dielectric Permittivity: Super Permittivity Under Extreme Confinement. J Phys Chem Lett 2020, 11, 10532-10537.

(47) It has been suggested ${ }^{5}$ that the anticorrelation of adjacent $z$ slices polarizations in confinement would be due to the negative correlation between neighboring water dipoles: our results show that neighboring water molecules are on average positively correlated as in the neat liquid; the polarization anticorrelation between adjacent $z$-slices arises 
from long-range anticorrelations between dipoles whose separation vector is orthogonal to the dipole orientation, a situation also present in the bulk. ${ }^{39}$

(48) Mouterde, T.; Keerthi, A.; Poggioli, A. R.; Dar, S. A.; Siria, A.; Geim, A. K.; Bocquet, L.; Radha, B. Molecular streaming and its voltage control in ångström-scale channels. Nature 2019, 567, 87-90.

(49) Jiménez-Ángeles, F.; Harmon, K. J.; Nguyen, T. D.; Fenter, P.; Olvera de la Cruz, M. Nonreciprocal interactions induced by water in confinement. Phys Rev Research 2020, 2, 043244 .

(50) Liu, T.; Serrano, J.; Elliott, J.; Yang, X.; Cathcart, W.; Wang, Z.; He, Z.; Liu, G. Exceptional capacitive deionization rate and capacity by block copolymer-based porous carbon fibers. Sci Adv 2020, 6, eaaz0906.

(51) Thompson, W. H. Solvation dynamics and proton transfer in nanoconfined liquids. Annu. Rev. Phys. Chem. 2011, 62, 599-619.

(52) Wei, Z.; Li, Y.; Cooks, R. G.; Yan, X. Accelerated Reaction Kinetics in Microdroplets: Overview and Recent Developments. Annu Rev Phys Chem 2020, 71, 31-51.

(53) Sato, T.; Sasaki, T.; Ohnuki, J.; Umezawa, K.; Takano, M. Hydrophobic Surface Enhances Electrostatic Interaction in Water. Phys Rev Lett 2018, 121, 206002.

(54) Parez, S.; Předota, M.; Machesky, M. Dielectric Properties of Water at Rutile and Graphite Surfaces: Effect of Molecular Structure. J. Phys. Chem. C 2014, 118, 4818-4834. 
Supporting Information

\title{
Confined Water's Dielectric Constant
}

\section{Reduction Is Due to the Surrounding Low}

\section{Dielectric Media and Not to Interfacial}

\section{Molecular Ordering}

\author{
Jean-François Olivieri, ${ }^{\dagger}$ James T. Hynes, ${ }^{\dagger, \ddagger}$ and Damien Laage*,† \\ $\dagger$ †ASTEUR, Department of Chemistry, École normale supérieure, PSL University, \\ Sorbonne Université, CNRS, 75005 Paris, France \\ $\ddagger$ Department of Chemistry, University of Colorado, Boulder, CO 80309, USA \\ E-mail: damien.laage@ens.psl.eu
}




\section{Simulations}

We use classical molecular dynamics to simulate a slab of liquid water between two parallel single sheet graphene plates. The two rigid graphene sheets are constructed from an ideal hexagonal lattice with a $1.42 \AA$ distance between neighboring carbon atoms. Four simulation boxes with different sizes and distances between the graphene sheets are employed. The

respective $x \times y \times z$ dimensions are $49.19 \times 51.12 \times 50 \AA^{3}$ for system $a, 81.16 \times 80.94 \times 80 \AA^{3}$ for system $b, 81.16 \times 80.94 \times 15 \AA^{3}$ for system $c$, and $110.68 \times 110.76 \times 110 \AA^{3}$ for system $d$. The number of water molecules in each system was determined iteratively via an insertion/deletion procedure such that the density in the middle of the box equals the experimental liquid water density. Water is modelled by the widely employed SPC/E potential, ${ }^{1}$ which has been shown ${ }^{2,3}$ to provide an excellent description of water static dielectric constant at ambient temperature. Graphene carbon Lennard-Jones parameters are taken from ref. 4 where they were optimized to reproduce DFT-based molecular dynamics simulation results. Long-range electrostatic interactions are described via the two-dimensional slab correction to the Ewald summation method. ${ }^{5}$ Water molecules are held rigid by the LINCS algorithm.

For each system, 2 to 4 independent initial configurations are prepared. Molecular dynamics simulations are run with Gromacs 2021. ${ }^{6}$ After equilibration at $300 \mathrm{~K}$, production runs are then propagated with a 2 -fs timestep with the velocity rescaling thermostat, ${ }^{7}$ and a 1-ps trajectory output interval. The respective simulation lengths for each system are $4.5 \mu \mathrm{s}$ for system $a, 400 \mathrm{~ns}$ for $b, 200 \mathrm{~ns}$ for $c$, and $150 \mathrm{~ns}$ for $d$.

A $80 \times 80 \times 80 \AA^{3}$ neat water reference system was prepared at the experimental liquid water density. The trajectories are propagated for 50 ns with the same procedure except for the three-dimensional periodic boundary conditions. Two different electrostatic boundary conditions are employed for the Ewald sum, embedding the system within a dielectric continuum of dielectric constant $\epsilon^{\prime}$, with respectively $\epsilon^{\prime}=\infty$ (tinfoil conditions, zero macroscopic field $\boldsymbol{E}=0$ ) and $\epsilon^{\prime}=0$ (zero electric displacement $\left.\boldsymbol{D}=0\right) .^{8}$ 


\section{Partially angle-averaged dipole-dipole interaction}

We consider the interaction between dipoles $\boldsymbol{\mu}_{1}$ and $\boldsymbol{\mu}_{2}$, using the notations of Fig. S1. The interaction energy is ${ }^{9}$

$$
u=-\frac{\mu_{1} \mu_{2}}{r^{3}}\left(2 \cos \theta_{1} \cos \theta_{2}-\sin \theta_{1} \sin \theta_{2} \cos \phi\right) .
$$

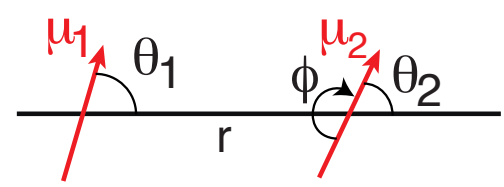

Figure S1: Dipole-dipole interaction.

For a fixed $\theta_{1}$ angle between dipole $\boldsymbol{\mu}_{1}$ and the axis between the two dipoles, the dipoledipole correlation averaged over the $\boldsymbol{\mu}_{2}$ orientations is

$$
\left\langle\boldsymbol{\mu}_{1} \cdot \boldsymbol{\mu}_{2}\right\rangle_{r, \theta_{1}}=\frac{\int_{0}^{\pi} \mathrm{d} \theta_{2} \sin \theta_{2} \int_{0}^{2 \pi} \mathrm{d} \phi \boldsymbol{\mu}_{1} \cdot \boldsymbol{\mu}_{2} e^{-\beta u\left(\theta_{1}, \theta_{2}, \phi, r\right)}}{\int_{0}^{\pi} \mathrm{d} \theta_{2} \sin \theta_{2} \int_{0}^{2 \pi} \mathrm{d} \phi e^{-\beta u\left(\theta_{1}, \theta_{2}, \phi, r\right)}} .
$$

A first-order perturbative expansion with respect to ratio between the dipole-dipole interaction potential and the thermal energy yields

$$
\left\langle\boldsymbol{\mu}_{1} \cdot \boldsymbol{\mu}_{2}\right\rangle_{r, \theta_{1}} \simeq \beta \mu_{1}^{2} \mu_{2}^{2} \frac{3 \cos ^{2} \theta_{1}-1}{3 r^{3}}
$$




\section{Kirkwood function in the slab}

Figure S2 shows the box size effect on the Kirkwood function $G_{K}^{\perp}(r ; z)$ eq. 6 : comparing the results obtained with $110.68 \times 110.76 \times 110 \AA^{3}$ and $81.16 \times 80.94 \times 80 \AA^{3}$ boxes shows that the dramatic drop in the short-range $(r \lesssim 20 \AA)$ part of the Kirkwood function responsible for the dielectric constant reduction is independent of the simulation box size in this range of sizes.

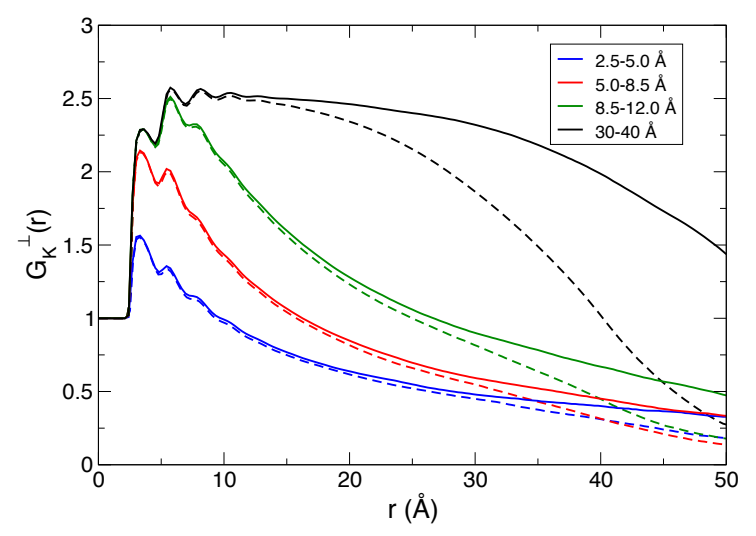

Figure S2: Kirkwood function $G_{K}^{\perp}(r ; z)$ eq. 6, calculated at increasing distances $z$ from the graphene wall in simulation boxes of $110.68 \times 110.76 \times 110 \AA^{3}$ (solid lines) and $81.16 \times 80.94 \times 80 \AA^{3}$ (dashes).

At large $r$ distances, it has been shown ${ }^{10}$ that the Kirkwood function for a homogeneous dipolar liquid includes a term due to the electrostatic boundary conditions that scales with ratio of the volume $v(r)$ of the sphere of radius $r$ 's fraction that remains within the simulation box and the total volume of the simulation box $V=L_{x} \times L_{y} \times L_{z}$. In the slab, at a distance $z$ from the wall, the volume $v(r)$ of the sphere's fraction that lies within the simulation box is

$$
\begin{aligned}
v(r)= & \frac{4 \pi}{3} r^{3} \text { for } \mathrm{r} \leq \mathrm{z} \\
& \frac{2 \pi}{3} r^{3}+\pi r^{2} z-\frac{\pi}{3} z^{3} \text { for } \mathrm{z}<\mathrm{r}<\mathrm{L}_{\mathrm{z}} / 2 .
\end{aligned}
$$


In the slab, we approximate the long-range decay by a term that scales with $v(r)$. Figure S3 shows the long-range decay of the Kirkwood function and the fit with eq. S4.

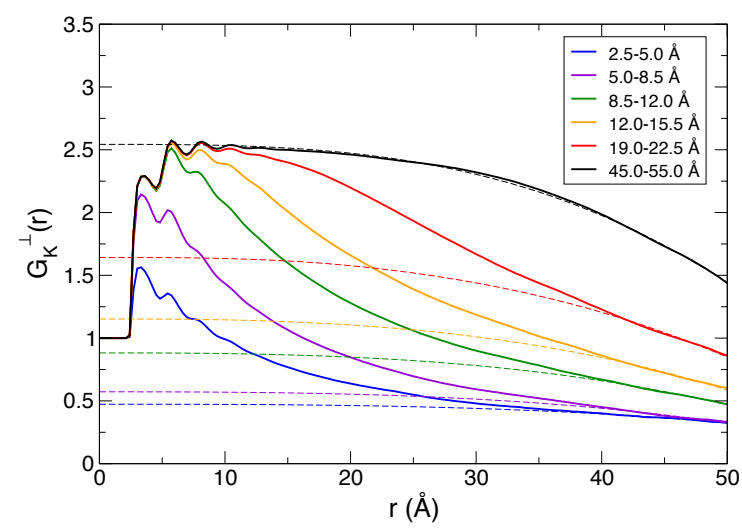

Figure S3: Kirkwood function $G_{K}^{\perp}(r ; z)$ eq. 6 , calculated at increasing distances $z$ from the graphene wall in the $110.68 \times 110.76 \times 110 \AA^{3}$ simulation box together with the long-range fit with eq. S4 (dashes).

For a dipolar liquid next to a wall, in the absence of orientational restrictions induced by the wall, the average correlation between the components perpendicular to the wall of a dipole $i$ at a distance $z$ from the wall and all the surrounding dipoles within a sphere of radius $R$ is determined from eq. S3 to be proportional to

$$
\left\langle\mu_{\perp}^{(i)} M_{z}^{(i)}(R)\right\rangle /\left\langle\left(\mu_{\perp}^{(i)}\right)^{2}\right\rangle \propto \int_{0}^{R} \mathrm{~d} r r^{2} \int_{\theta_{\min }(r, z)}^{\pi} \mathrm{d} \theta \sin \theta \frac{3 \cos ^{2} \theta-1}{r^{3}}
$$

where the $\theta$ angle between the dipole pair direction and the direction normal to the wall is integrated over the $\left[\theta_{\min }(r, z), \pi\right]$ interval, with $\theta_{\min }(r, z)=0$ for $r \leq z$ and $\theta_{\min }(r, z)=$ $\arccos (z / r)$ for $r>z$. This leads to

$$
\begin{aligned}
\left\langle\mu_{\perp}^{(i)} M_{z}^{(i)}(R)\right\rangle /\left\langle\left(\mu_{\perp}^{(i)}\right)^{2}\right\rangle & =0 \text { for } \mathrm{R} \leq \mathrm{z} \\
& \propto-\frac{2}{3}+\frac{z}{R}\left[1-\frac{1}{3}\left(\frac{z}{R}\right)^{2}\right] \quad \text { for } \mathrm{R}>\mathrm{z}
\end{aligned}
$$

Figure S4 shows that eq. S6 provides a good description of the corrected Kirkwood function 
$G_{K c}^{\perp}(r ; z)$ long-range decay to its plateau value.

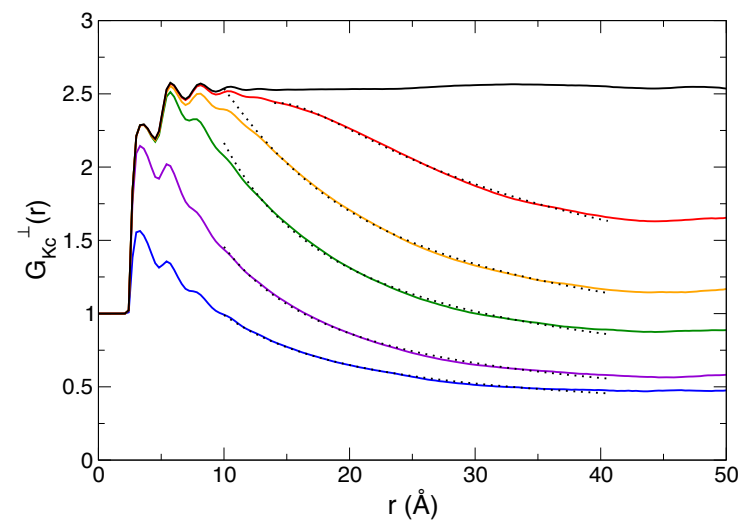

Figure S4: Kirkwood function $G_{K c}^{\perp}(r ; z)$ corrected for the long-range decay (eq. 8) at the same distances $z$ from the graphene wall as shown in Fig. S3 in the $110.68 \times 110.76 \times 110 \AA^{3}$ simulation box together with the fits with eq. S6 (dashes).

Figure S5 shows the angle- and distance-dependent Kirkwood function $G_{K}^{\perp}(r, \cos \theta ; z)-$ such that $G_{K}^{\perp}(r ; z)=\int_{-1}^{1} \mathrm{~d} \cos \theta G_{K}^{\perp}(r, \cos \theta ; z)$ - in the middle of the slab, in the first and second layers and their respective differences with the middle of the slab. Panel a exhibits the angular dependence expected for a dipolar liquid with positive correlations found for dipoles located along the normal vector and negative correlations in the plane parallel to the wall, together with some short-range fluctuations along $r$ due to the liquid structure. Panels b and c display similar features but reveal the excluded volume regions due to the wall for $\cos \theta>0$ (see also Fig. 3d). The differences of the first and second layers from the middle layer shown in panels $\mathrm{d}$ and e reveal that the decreased average dipole correlation between the wall vicinity and the middle of the slab originates essentially from the water molecules that have been excluded from the positive correlation region by the wall. Smaller additional effects arise from the anisotropic orientational distribution of water molecules in the first shell, which decreases the average dipole component along the normal direction with respect to its isotropic value in the middle of the slab. 

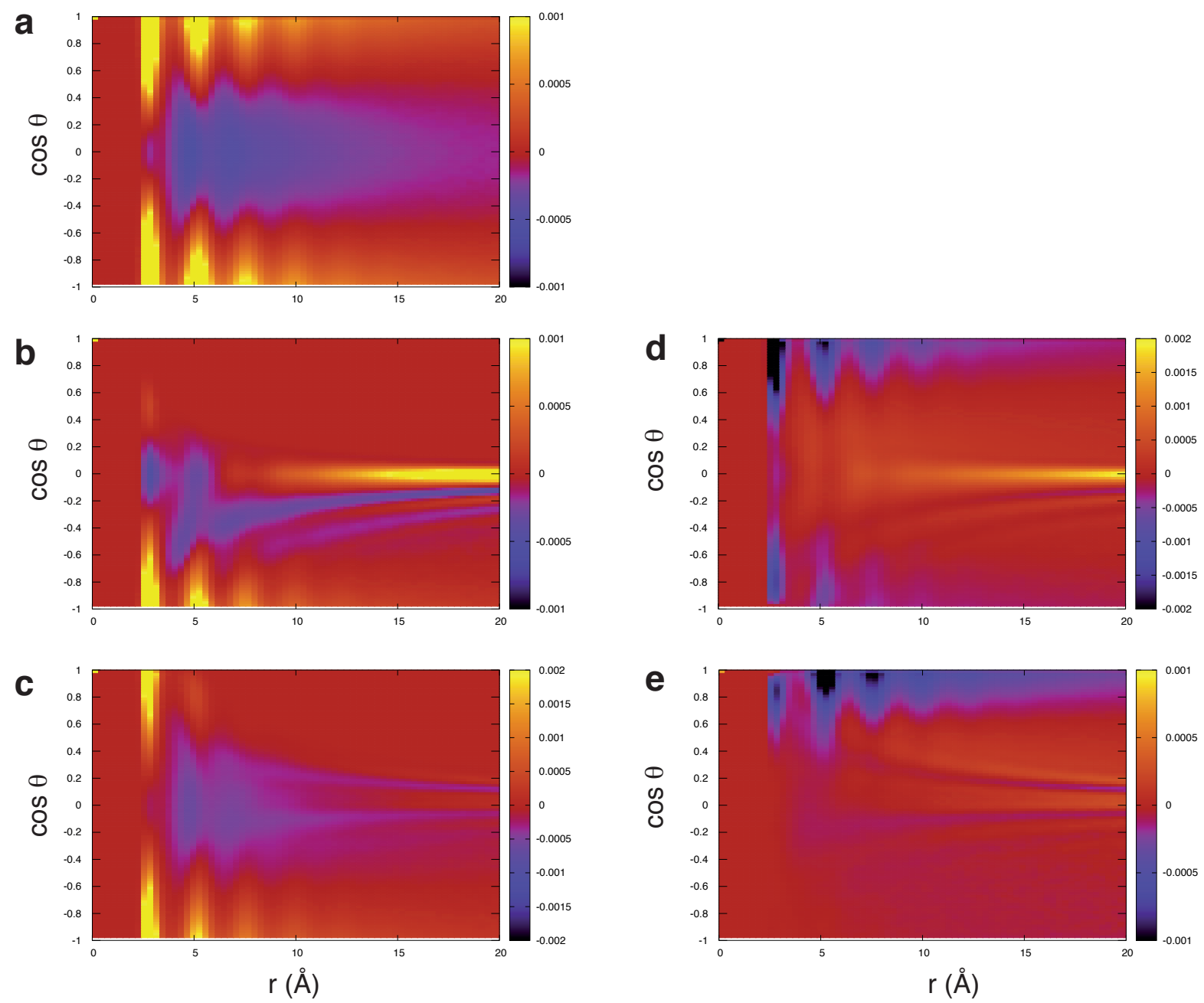

Figure S5: Kirkwood function $G_{K}^{\perp}(r, \cos \theta ; z)$ calculated in the $110.68 \times 110.76 \times 110 \AA^{3}$ simulation box a) in the middle of the box $(45<z<55 \AA)$, b) in the first layer $(2.5<z<5.0 \AA)$, and c) in the second layer $(5.0<z<8.5 \AA)$; d) difference between the $G_{K}^{\perp}(r, \cos \theta ; z)$ values determined in the first layer and in the middle of the box, e) idem between the second layer and the middle of the box. The $\theta$ angle is defined with respect to the normal vector pointing towards the wall. 


\section{Local parallel dielectric constant $\epsilon_{\|}(z)$}

The component of the local dielectric constant parallel to the graphene plates at a distance $z$ from the wall is ${ }^{11}$

$$
\epsilon_{\|}(z)=1+2 \pi \beta\left[\left\langle\mathbf{m}_{\|}(z) \mathbf{M}_{\|}\right\rangle-\left\langle\mathbf{m}_{\|}(z)\right\rangle\left\langle\mathbf{M}_{\|}\right\rangle\right]
$$

where $\mathbf{M}_{\|}$is the $x y$ component of the total water dipole along the direction parallel to the graphene planes, $\beta=1 /\left(k_{B} T\right)$, and $\mathbf{m}_{\|}(z)$ is the local polarization density along the $x y$ plane, averaged in the plane parallel to the graphene wall and calculated with the procedure described in ref. 12. The $\epsilon_{\|}(z)$ profile is shown in Fig. S6 for a $50 \AA$ separation between the graphene plates. The parallel dielectric constant local enhancement at the graphene interface is similar to the results recently reported in refs. 13-15.

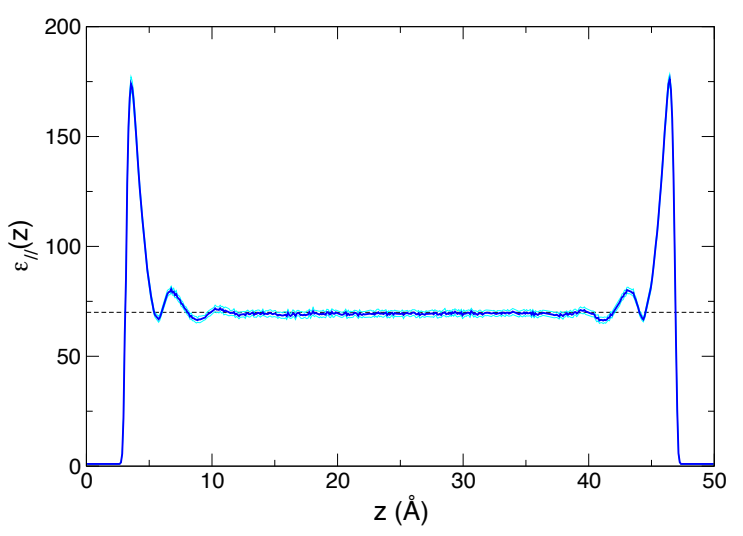

Figure S6: Local dielectric constant profile $\epsilon_{\|}(z)$ across a $50 \AA$-thick confined water slab (eq. S7) together with the $95 \%$ confidence interval.

In analogy to eq. S5, for a dipolar liquid next to a wall, the average correlation between the components parallel to the wall of a dipole $i$ at a distance $z$ from the wall and all the surrounding dipoles within a sphere of radius $R$ is proportional to

$$
\left\langle\mu_{\|}^{(i)} M_{\|}^{(i)}(R)\right\rangle /\left\langle\left(\mu_{\|}^{(i)}\right)^{2}\right\rangle \propto \int_{z}^{R} \mathrm{~d} r r^{2} \int_{0}^{\pi} \mathrm{d} \theta \sin \theta \frac{1}{2 \pi} \int_{\phi_{\min }(r, z, \theta)}^{2 \pi-\phi_{\min }(r, z, \theta)} \frac{3 \cos ^{2} \theta-1}{r^{3}}
$$


where $\phi_{\min }(r, z, \theta)$ is defined by $\phi_{\min }=\arccos [z /(r \sin \theta)]$ for $r \sin \theta>z$ and $\phi_{\min }=0$ otherwise.

Figure S7 shows that the interface excluded volume effect is smaller in magnitude for the parallel component than for the perpendicular one. In the parallel case, when $R$ slightly exceeds the distance $z$ to the wall, the latter excludes molecular dipoles that would bring a negative correlation in the bulk (see the schematic representation of the dipolar electric field produced by a dipole parallel to the wall in Fig. S8), which thereby induces an enhancement of the dielectric constant. For larger $R$ radii, both negatively and positively correlated molecules are excluded by the wall, and the effect on the dielectric constant reaches a plateau value.

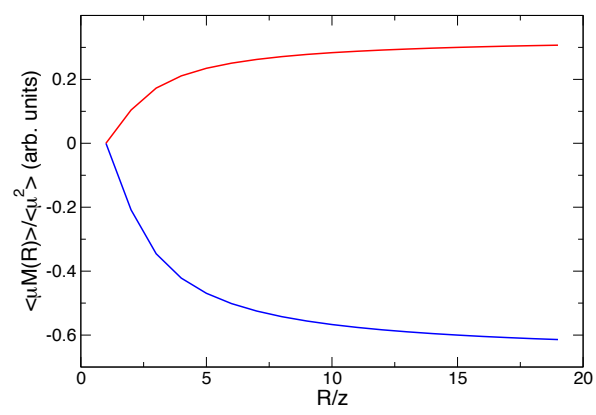

Figure S7: Wall excluded-volume effect on the average correlations of the parallel (red) and perpendicular (blue) dipole components for increasing $R$ radii (eqs. S5,S8).

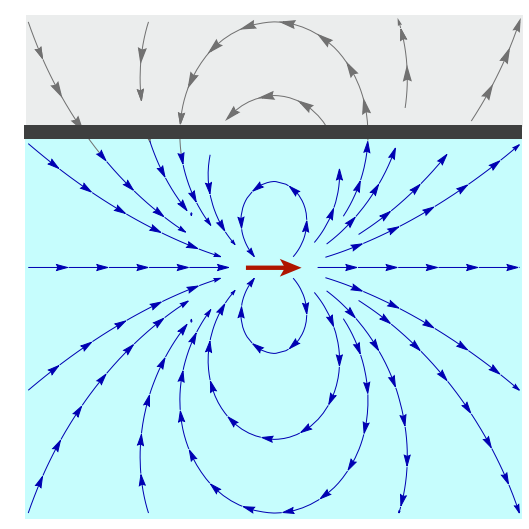

Figure S8: Schematic representation of the dipolar electric field produced by a dipole parallel to the wall 


\section{References}

(1) Berendsen, H. J. C.; Grigera, J. R.; Straatsma, T. P. The missing term in effective pair potentials. J Phys Chem 1987, 91, 6269-6271.

(2) Wu, Y.; Tepper, H. L.; Voth, G. A. Flexible simple point-charge water model with improved liquid-state properties. J Chem Phys 2006, 124, 024503.

(3) Fennell, C.; Li, L.; Dill, K. Simple liquid models with corrected dielectric constants. J Phys Chem B 2012, 116, 6936-6944.

(4) Rana, M.; Chandra, A. Ab initio and classical molecular dynamics studies of the structural and dynamical behavior of water near a hydrophobic graphene sheet. J Chem Phys 2013, 138, 204702.

(5) Yeh, I.-C.; Berkowitz, M. L. Ewald summation for systems with slab geometry. J Chem Phys 1999, 111, 3155-3162.

(6) Abraham, M. J.; Murtola, T.; Schulz, R.; Páll, S.; Smith, J. C.; Hess, B.; Lindahl, E. GROMACS: High performance molecular simulations through multi-level parallelism from laptops to supercomputers. SoftwareX 2015, 1, 19-25.

(7) Bussi, G.; Donadio, D.; Parrinello, M. Canonical sampling through velocity rescaling. J Chem Phys 2007, 126, 014101.

(8) Zhang, C.; Sprik, M. Computing the dielectric constant of liquid water at constant dielectric displacement. Phys Rev B 2016, 93, 144201.

(9) Israelachvili, J. N. Intermolecular and Surface Forces; Academic Press, 2011.

(10) Caillol, J. Asymptotic behavior of the pair-correlation function of a polar liquid. J Chem Phys 1992, 96, 7039-7053. 
(11) Ballenegger, V.; Hansen, J. Dielectric permittivity profiles of confined polar fluids. $J$ Chem Phys 2005, 122, 114711.

(12) Schlaich, A.; Knapp, E. W.; Netz, R. R. Water Dielectric Effects in Planar Confinement. Phys Rev Lett 2016, 117, 048001.

(13) Motevaselian, M.; Aluru, N. Confinement-Induced Enhancement of Parallel Dielectric Permittivity: Super Permittivity Under Extreme Confinement. J Phys Chem Lett 2020, 11, 10532-10537.

(14) Ruiz-Barragan, S.; Muñoz-Santiburcio, D.; Körning, S.; Marx, D. Quantifying anisotropic dielectric response properties of nanoconfined water within graphene slit pores. Phys Chem Chem Phys 2020, 22, 10833-10837.

(15) Loche, P.; Ayaz, C.; Wolde-Kidan, A.; Schlaich, A.; Netz, R. R. Universal and NonUniversal Aspects of Electrostatics in Aqueous Nano-Confinement. J Phys Chem B 2020, 124, 4365-4371. 